INPLASY PROTOCOL

To cite: Cerón et al. Therapies for bruxism in dentistry: A systematic review protocol. Inplasy protocol 2021100080. doi:

10.37766/inplasy2021.10.0080

Received: 20 October 2021

Published: 21 October 2021

Corresponding author: Lissette Cerón

lissette.ceron@ucuenca.edu.ec

Author Affiliation:

Universidad de Cuenca

Facultad de Odontologia

Support: None.

Review Stage at time of this submission: Data analysis.

Conflicts of interest:

None declared.

\section{Therapies for bruxism in dentistry: A systematic review protocol}

Review question / Objective: The aim of this study is to evaluate the methodological quality of the literature and the risk of bias used in systematic reviews of therapies for bruxism in dentistry, applying the Amstar II qualitative guide and to answer the following question: What do we know so far about the different treatments applied for bruxism and their effectiveness, as well as what is the overall confidence of the systematic reviews evaluating this topic?

Condition being studied: There is an ongoing debate about the causal factors associated with patients diagnosed with bruxism and thus various treatment approaches, so according to the available scientific evidence there is no consensus on which is the most effective. (4) (8) (10). According to several studies, occlusal splints do not currently have a scientifically proven efficacy for the management of bruxism, because they lack randomized controlled clinical studies, and should therefore be considered as a limited treatment modality, since the effect of the splints does not seem to address the cause of bruxism and serves mainly for the management of the signs and symptoms of this disorder (11) (12). Alternative therapies such as relaxation and biofeedback have been proposed for bruxism, especially in cases of daytime bruxism, which are more related to stress and anxiety. (13). There are also studies that support the use of the NTI-tssa device can be used successfully, however, it may present side effects if necessary checks and readjustments are not performed (14). Some medications can be used to decrease bruxing episodes, but some pharmacological treatments may not be safe if used for prolonged periods of time, considering the inherent side effects or risks of dependence (15).

INPLASY registration number: This protocol was registered with the International Platform of Registered Systematic Review and Meta-Analysis Protocols (INPLASY) on 21 October 2021 and was last updated on 21 October 2021 (registration number INPLASY2021100080).

\section{INTRODUCTION}

Review question / Objective: The aim of this study is to evaluate the methodological quality of the literature and the risk of bias used in systematic reviews of therapies for bruxism in dentistry, applying the Amstar II qualitative guide and to answer the following question: What do we know so far about the different treatments applied for bruxism and their effectiveness, as well as 
what is the overall confidence of the systematic reviews evaluating this topic?

Rationale: There have been several investigations over time on the definition of bruxism. The latest consensus described it as a behavior or activity, forcefully maintaining a certain jaw position and forcefully pushing or moving the jaw forward or sideways, both activities without the necessary presence of contact with the teeth, and considered a disorder only when the patient develops signs and symptoms. (1) (2), as a result, different treatments have been developed to solve bruxism, such as: botulinum toxin type A (BoNT-A), occlusal splints, biofeedback, physiotherapy, pharmacotherapy; however, there is controversy about their effectiveness, and in addition, these studies are not always carried out meticulously and the risk of bias can lead to malpractice. (3) (4) (5) (6) (7) (8) (9). Indeed, as researchers, it is important for us to provide the most accurate and up-to-date evidence on bruxism therapies to facilitate the clinician's decision making.

Condition being studied: There is an ongoing debate about the causal factors associated with patients diagnosed with bruxism and thus various treatment approaches, so according to the available scientific evidence there is no consensus on which is the most effective. (4) (8) (10). According to several studies, occlusal splints do not currently have a scientifically proven efficacy for the management of bruxism, because they lack randomized controlled clinical studies, and should therefore be considered as a limited treatment modality, since the effect of the splints does not seem to address the cause of bruxism and serves mainly for the management of the signs and symptoms of this disorder (11) (12). Alternative therapies such as relaxation and biofeedback have been proposed for bruxism, especially in cases of daytime bruxism, which are more related to stress and anxiety. (13). There are also studies that support the use of the NTI-tssa device can be used successfully, however, it may present side effects if necessary checks and readjustments are not performed (14). Some medications can be used to decrease bruxing episodes, but some pharmacological treatments may not be safe if used for prolonged periods of time, considering the inherent side effects or risks of dependence (15).

\section{METHODS}

Search strategy: Cochrane ("systematic review" OR "systematic reviews" OR "systematic literature review" OR "systematic literature reviews" OR "Cochrane" OR "meta analysis" OR "meta synthesis" OR "systematic" OR "review" OR "reviews") AND ("bruxism" OR " awake bruxism" OR "sleep bruxism" OR "bruxist") AND ( "therapy" OR "diagnostic" OR "pain" OR "tooth wear" OR "mastication"OR "adult" OR "bite" OR "treatment" OR "effectiveness") n Title Abstract Keyword Scopus ("bruxism sleep" OR "awake bruxism OR "bruxism" AND "systematic review") Pubmed (""bruxism" "[MeSH Terms] OR ""sleep bruxism""[MeSH Terms] OR ""sleep bruxism""[MeSH Terms] OR ""awake bruxism""[Other Term]) AND ("'systematic review""[Publication Type] OR ""systematic reviews as topic" "[MeSH Terms] OR " "systematic review" "[All Fields] OR ((" "review" "[Publication Type] OR ""review literature as topic" "[MeSH Terms] OR " "review" "[AII Fields]) AND (" "publications" "[MeSH Terms] OR ""literature""[MeSH Terms])) OR ""review literature as topic" "[MeSH Terms])" GOOGLE SCHOLAR "treatment bruxism" AND "systematic review" Open Grey "treatment" AND "bruxism"” EMBASE \#1 = ('bruxism'/exp OR bruxism OR 'sleep bruxism'/exp OR 'sleep bruxism' OR 'awake bruxism') \#2 = ('systematic review' OR 'integrative review' OR 'meta-analysis' OR 'meta analysis' OR overview OR review OR 'systematic literature review' OR 'rapid review') \#3 = (\#1 AND \#2).

Participant or population: Studies involving adults (18 years of age and older) diagnosed as bruxers will be considered for this research. There will be no restriction of sex and ethnicity. 
Intervention: According to the literature, interventions for patients with bruxism are of wide variation and can be divided into the following groups: (1) intraoral interventions: occlusal adjustment, occlusal splints, mandibular advancement appliances, NTI (trigeminal nociceptive inhibitor) splint; (2) physiotherapy of the masticatory muscles with electrical stimulation: biofeedback, transcutaneous microcurrent stimulation, transcutaneous electrical nerve stimulation, contingent electrical stimulation; (3) pharmacotherapy: antidepressants, L-dopa inhibitors, antiepileptic drugs, sympatholytics, antihistamines or dopaminergics; (4) intramuscular injection: botulinum toxin $A$; (5) behavioral: relaxation techniques, "sleep hygiene" measures, cognitive treatment, psychological counseling.

\section{Comparator: None.}

Study designs to be included: We will include systematic literature reviews with or without meta-analysis.

Eligibility criteria: The included studies were SR with or without meta-analysis that evaluated the different treatments used in adult patients (18 years of age and older) diagnosed as bruxers; studies that include patients with tooth wear, temporomandibular disorders or orofacial pain are also eligible for inclusion. There is still no consensus on the definition of bruxism and thus there is much controversy about which treatment is most effective. Therefore, to address this concern, the authors considered including SRs reporting on all currently applied treatments for bruxism.No time or language restrictions were applied. The exclusion criteria included: 1. Literature reviews, intervention studies, observational studies, in vitro laboratory investigations, randomized controlled clinical studies, abstracts, commentaries, case reports, protocols, personal opinions, expert opinions, letters and posters. 2. Studies that did not meet the minimum criteria for SR proposed by the Cochrane Handbook for SR of Interventions. 4. The required data are not available after three attempts within a 30 - day interval to contact the corresponding author by e-mail. 5. SR whose treatment effectiveness results are not directly related to bruxism.

Information sources: Electronic database: • PUBMED - SCOPUS - COCHRANE • EMBASE - LILACS Gray Literature • Google Scholar - OpenGrey.

Main outcome(s): Decreased electromyographic activity in patients diagnosed with bruxism.

Additional outcome(s): None.

Data management: The protocol for this systematic review review will be written in accordance with PRISMA-P (Preferred Reporting Items for Systematic Review and Meta-Analysis Protocols). This protocol will be registered in the INPLASY database (international platform of registered systematic review and meta-analysis protocols). Two independent and calibrated reviewers ( $L$ and $M$ ) will perform electronic and manual bibliographic searches, in duplicate in several databases, including PUBMED; SCOPUS; COCHRANE; EMBASE databases, for systematic reviews until October 2020 without language restriction. Reviewers will independently extract data from systematic reviews, the results of this pre-screening will be discussed in rounds and disagreements will be resolved by discussion until a consensus is reached. A third author (W.B) will verify the accuracy of all articles. Disagreements will also be resolved by consensus among all reviewers. Software tools will be used to perform data extraction and statistical analyses.

Quality assessment / Risk of bias analysis: The AMSTAR II tool will be used to assess the risk of bias.

Strategy of data synthesis: The synthesized data are as follows: Systematic reviews that meet the inclusion criteria. The data will be summarized as follows: decrease in electromyographic activity depending on the treatment applied in patients confirmed with a diagnosis of bruxism. The formal 
method of combining data from individual studies will be a narrative synthesis, relating to each outcome and follow-up time. Color charts will be constructed, illustrating whether that study showed better results for the test group or the control group. A quantitative synthesis is not planned.

Subgroup analysis: None.

Sensitivity analysis: None.

Language: No language restriction.

\section{Country(ies) involved: Ecuador.}

Other relevant information: References - 1. 1. Lobbezoo F et al. International consensus on the assessment of bruxism: Report of a work in progress. $J$ Oral Rehabil. 2018. 2. Raphael KG et al. Is bruxism a disorder or a behaviour? Rethinking the international consensus on defining and grading of bruxism. Journal Oral Rehabilitation. 2016. 3. Sendra LA et al. Clinical outcomes of botulinum toxin type $A$ injections in the management of primary bruxism in adults: A systematic review. J Prosthet Dent. 2021. 4. De la Torre Canales G CSMdACGRMD. Is there enough evidence to use botulinum toxin injections for bruxism management? A systematic literature review. Clin Oral Investig. 2017. 5. Riley $\mathbf{P}$ et al. Oral splints for temporomandibular disorder or bruxism: a systematic review. Br Dent Journal. 2020. 6. Jokubauskas L et al. Oral appliances for managing sleep bruxism in adults: a systematic review from 2007 to 2017 . J Oral Rehabil. 2018. 7. Jokubauskas $L$ et al. Efficacy of biofeedback therapy on sleep bruxism: A systematic review and metaanalysis. J Oral Rehabil. 2018. 8. Amorim CSM et a. Effect of Physical Therapy in Bruxism Treatment: A Systematic Review. J Manipulative Physiol Ther. 2018. 9. Macedo CR et al. Pharmacotherapy for sleep bruxism. Cochrane Database Syst Rev. 2014. 10. Fernández-Núñez T AMSGEC. Efficacy of botulinum toxin in the treatment of bruxism: Systematic review. Med Oral Patol Oral Cir Bucal. 2019. 11. Dao TT et al. Oral splints: the crutches for temporomandibular disorders and bruxism? Crit Rev Oral Biol Med. 1998.

12. Macedo $C R$ et al. Occlusal splints for treating sleep bruxism (tooth grinding). Cochrane Database Syst Rev. 2007. 13. Wang LF et al. Biofeedback treatment for sleep bruxism: a systematic review. Sleep Breath. 2014. 14. Stapelmann H TJ. The NTI-tss device for the therapy of bruxism, temporomandibular disorders, and headache - where do we stand? A qualitative systematic review of the literature. BMC Oral Health. 2008. 15. Huynh NT et al. Comparison of various treatments for sleep bruxism using determinants of number needed to treat and effect size. Int J Prosthodont. 2006.

Keywords: Bruxism, adult sleep bruxism, waking bruxism, parafunctional habits, bruxism treatment, systematic reviews.

Dissemination plans: Publication in a high impact journal to disseminate the results of our overview and in national and international congresses.

Contributions of each author:

Author 1 - Lissette Cerón - Introduction writing, protocol writing, data collection, data analysis.

Email: lissette.ceron@ucuenca.edu.ec

Author 2 - Mishelle Pacheco - Search strategy, protocol drafting, materials and methods drafting, data collection, data analysis.

Email: mishelle.pacheco@ucuenca.edu.ec Author 3 - Bolivar Delgado - Search strategy, Supervisor.

Email: bolivar.delgado@ucuenca.edu.ec Author 4 - Wilson Bravo - Conceiving the review, search strategy, analysis of data. Email: wilson.bravo@cuenca.edu.ec 